\title{
Nothing's impossible
}

Proofs of what is impossible, as John Bell once remarked, often demonstrate little more than their authors' own lack of imagination. Bell said this in discussion of one of the most famous, and famously erroneous, impossibility proofs of all time, one constructed in 1932 by Hungarian physicist John von Neumann. It purported to show that no hidden-variables interpretation of quantum theory - one attributing definite trajectories and other objective properties to quantum systems that might account deterministically for experimental outcomes - could possibly reproduce all the predictions of quantum theory.

Of course, von Neumann certainly had more than a little imagination, although this was perhaps not the only time it showed its limitations. (In the 1950s, he strongly advised US authorities on the wisdom of an immediate unilateral nuclear attack on the Soviet Union, apparently because he could see no conceivable way that the two nations might escape eventual mutual annihilation.) In any event, Bell's phrase does capture a truth - that impossibility proofs really demonstrate only what is inconceivable to one mind, or one set of minds, which may (for all their other wonderful capacities) be blinkered. Such traps, as history illustrates, afflict us far more frequently than we expect.

Von Neumann's mistake was subtle enough - and on a topic on which most physicists were already convinced - that it stood as received wisdom, beyond questioning, for over two decades. He started from five assumptions that, he thought, any hidden-variables interpretation of quantum theory would clearly have to satisfy. To most physicists, these assumptions seem eminently plausible.

His fifth and final assumption asserted that the expectation values of summed variables, evaluated for example in experiments on a set of systems prepared so that all the hidden variables are identical, should satisfy the algebraic relation,

$\langle\mathrm{X}+\mathrm{Y}\rangle=\langle\mathrm{X}\rangle+\langle\mathrm{Y}\rangle$ : the average of a sum of two variables should equal the sum of the averages of those variables.

This does indeed seem plausible, at least naively. It seems hard to get your head moving towards any conceptual space where

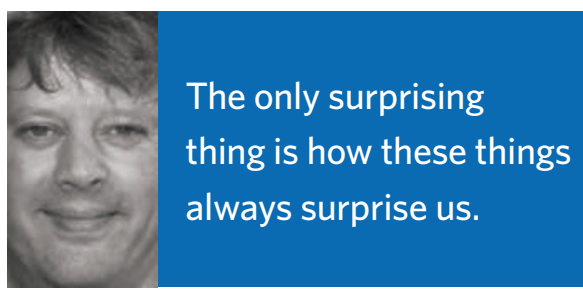

this condition might not be true. It took Bell's mental dexterity and perseverance to clarify how totally inappropriate was von Neumann's use of this condition. The assumption rests on the idea of socalled 'non-contextuality' - that physical quantities can be thought of as having values completely independently of the devices used to measure them.

Of course any classical theory would work this way. But contextuality is precisely one of the most interesting things about quantum theory, and one of the things that any hidden-variable theory needs to capture. Any such theory worth its salt has to allow the action of experimental devices to help determine the values of a system's variables. This, Bell showed, makes von Neumann's assumptions completely out of place "absurd", as he put it.

This example illustrates the general problem - the inherent weakness of such proofs to hidden assumptions, because such assumptions are sometimes so difficult to see amidst the conceptual debris surrounding them. Proofs have loopholes that are effectively invisible to their authors. It is as true today as it was four centuries ago.

In 1651, an Italian Jesuit Giovanni Battista Riccioli published a book entitled Almagestum Novum, in which he gave seventy-seven proofs of the impossibility of the Earth's motion, in particular its rotation. Riccioli's book was written against the Copernicans, and he was no fool. In one historian's view, Riccioli produced "the lengthiest, most penetrating, and authoritative analysis of the question of Earth's mobility or immobility made by any author of the sixteenth and seventeenth centuries."

Riccioli's proofs were, I expect, just as convincing to him and many of his contemporaries as von Neumann's proofs about quantum theory were to physicists of the latter twentieth century. As Christopher Graney points out in a paper that has made Riccioli's writings available from the original Latin text (arxiv:10113778), only two of the seventy-seven proofs are obviously religious. Most make eminently good sense given the empirical knowledge of the day.

"If Earth had a diurnal rotation", one proof observes, "then heavy bodies falling near the equator would have a fundamentally different motion than identical bodies falling near the poles under identical conditions." Similarly, "Heavy bodies launched perpendicularly upwards fall back upon the location from which they were launched. If the Earth had diurnal and annual motions, these bodies would follow curved trajectories." Indeed, Riccioli was right - it's only that the weakness of the Coriolis force he clearly envisaged meant that it hadn't yet been measured in 1651 .

Another of his observations runs along the same lines: "If Earth moves, then the clouds and the birds in the air would be seen to fly west, as they were left behind by the Earth." Riccioli was only making an implicit assumption about the magnitude of this effect. Again, a hidden presumption, later revealed. The only surprising thing is how these things always surprise us.

In recent times, we've seen a string of proofs establishing the alleged impossibility of breaking the security of various schemes for quantum cryptography. Yet each progressively more intricate scheme only seems to inspire counter efforts that identify new loopholes. Perhaps the only impossibility proofs that really work are constructive proofs that actually establish positive results. For example, David Bohm's 1952 alternative formulation of quantum theory gave a proof by explicit construction that von Neumann's argument couldn't possibly be correct; indeed, that no argument could establish the impossibility of a consistent deterministic interpretation of the non-locality inherent in quantum theory; after all, Bohm had produced one. It couldn't then be proved out of existence.

I'm sure this is a lesson humanity will be learning for centuries to come. "All historical experience", as Max Weber wrote, "confirms that men might not achieve the possible if they had not, time and time again, reached out for the impossible."

MARK BUCHANAN 